\title{
On Commutative Properties of Halftoning and Their Applications
}

\author{
Y. B. Karasik \\ School of Computer Science, Carleton University, \\ 1125 Colonel By Drive, Ottawa, Canada K1S 5B6 \\ and \\ Computer Science Department, Tel Aviv University, Israel \\ e-mail: karasik@turing.scs.carleton.ca.or karasik@math.tau.ac.il
}

\begin{abstract}
We investigate relations between halftoning and the binary image algebra which includes set and morphological operations. We show that halftoning has important commutative properties with respect to unions, intersections, Minkowski additions and subtractions. Based on this fact a novel approach to implementation of 3-dimensional optical image processing through manipulating 2-dimensional images is proposed.
\end{abstract}

\section{Introduction}

The halftoning technique, which converts a gray scale image into a binary image, is an important tool for image encoding and processing.

First, it is aimed at rendering continuous tone images on devices such as laser printers where only two levels (usually black and white) can be represented at any point. In such devices, a continuous gray scale image, whose gray tone varies across the plane as $\operatorname{gray}(x, y)$, is encoded as a discrete binary image consisting of black dots of varying sizes located at the nodes $\{(i h, j h)\}_{i, j=-N}^{N}$ of a lattice having a spacing $h$, so that the size of a dot at a point $(x, y)$ is proportional to the value $\operatorname{gray}(x, y)$. Such a halftone image when viewed at normal reading distance gives the effect of a continuous tone image.

Besides image encoding, halftoning has also turned out to be an important technique for nonlinear image processing. For example, in conjuction with a coherent optical system, it was used for analog-to-digital conversion [L], and for a variety of other nonlinear transformations on images [KG, DS].

However, there exists another powerful tool for image processing, namely: image algebra [HJS] whose connection with halftoning has not yet been properly investigated. The purpose of the present paper is to bridge this gap and to show that halftoning has important commutative properties with respect to basic set and morphological operations such as union $(\bigcup)$, intersection $(\bigcap)$, dilation $(+)$, and erosion (-). It is investigated more closely in the section that follows. 


\section{Commutative properties of halftoning}

Let $f_{X}(x, y)$ be the gray tone distribution of an image $X$ across the plane. Then we assume that

$$
f_{A \cup B}(x, y)=\max \left\{f_{A}(x, y), f_{B}(x, y)\right\},
$$

and

$$
f_{A \cap B}(x, y)=\min \left\{f_{A}(x, y), f_{B}(x, y)\right\}
$$

respectively.

Based on these assumptions, we can now state the following two lemmas:

Lemma 1. Let $H T(X)$ be the halftone representation of an image $X$.

Then the following relationships hold:

$$
\begin{aligned}
& H T(A \cup B)=H T(A) \cup H T(B) ; \\
& H T(A \cap B)=H T(A) \cap H T(B) .
\end{aligned}
$$

Proof. Let $d[x, y, R]$ be a dot of the size $R$ located at the point $(x, y)$ which satisfies the relationship

$$
d\left[x, y, R_{1}\right]+d\left[x, y, R_{2}\right]=d\left[x, y, R_{1}+R_{2}\right] .
$$

For example, it can be a circular or rectangular dot.

Obviously,

$$
\begin{aligned}
& d\left[x, y, R_{1}\right] \cup d\left[x, y, R_{2}\right]=d\left[x, y, \max \left\{R_{1}, R_{2}\right\}\right] ; \\
& d\left[x, y, R_{1}\right] \cap d\left[x, y, R_{2}\right]=d\left[x, y, \min \left\{R_{1}, R_{2}\right\}\right] .
\end{aligned}
$$

Since,

$$
H T(A)=\bigcup_{i j} d\left[i h, j h, f_{A}(i h, j h)\right]
$$

and

$$
H T(B)=\bigcup_{i j} d\left[i h, j h, f_{B}(i h, j h)\right]
$$

then we have:

$$
\begin{gathered}
H T(A) \cup H T(B)=\bigcup_{i j}\left\{d\left[i h, j h, f_{A}(i h, j h)\right] \cup d\left[i h, j h, f_{B}(i h, j h)\right]\right\}= \\
\bigcup_{i j} d\left[i h, j h, \max \left\{f_{A}(i h, j h), f_{B}(i h, j h)\right\}\right] .
\end{gathered}
$$

Let $z=f_{A \cup B}(x, y)$ be the equation of the surface bounding $A \cup B$. Obviously,

$$
f_{A \cup B}(x, y)= \begin{cases}f_{A}(x, y) & \text { if } f_{A}(x, y) \geq f_{B}(x, y) \\ f_{B}(x, y) & \text { otherwise. }\end{cases}
$$


Hence,

$$
f_{A \cup B}(x, y)=\max \left\{f_{A}(x, y), f_{B}(x, y)\right\}
$$

and we can conclude that

$$
\begin{gathered}
\bigcup_{i j} d\left[i h, j h, \max \left\{f_{A}(i h, j h), f_{B}(i h, j h)\right\}\right]= \\
\bigcup_{i j} d\left[i h, j h, f_{A \cup B}(x, y)\right]=H T(A \cup B) .
\end{gathered}
$$

Thus, we obtain that

$$
H T(A) \cup H T(B)=H T(A \cup B) .
$$

The proof that

$$
H T(A \cap B)=H T(A) \cap H T(B)
$$

is analogous.

Lemma 2. Let $\approx$ A be a step approximation of the image $A$, i.e. $f_{\approx}(x, y)=$ $f_{A}(i h, j h)$ if $x \in(i h-h / 2, i h+h / 2]$ and $y \in(j h-h / 2, j h+h / 2]$. Then the following relationship holds:

$$
H T(\widetilde{\tilde{A}}+\widetilde{\widetilde{B}})=H T(\widetilde{\tilde{A}})+H T(\widetilde{B})=H T(A)+H T(B)
$$

Proof. Due to the definitions introduced in the proof of the previous lemma, we have:

$$
H T(\widetilde{\tilde{A}}+\widetilde{\widetilde{B}})=\bigcup_{m n} d\left[m h, n h, \max _{\substack{x_{1}+x_{2}=m h \\ y_{1}+y_{2}=n h}}\left(f_{\approx}\left(x_{1}, y_{1}\right)+f_{\widetilde{B}}\left(x_{2}, y_{2}\right)\right)\right] .
$$

Let $x_{1} \in(i h-h / 2, i h+h / 2], y_{1} \in(j h-h / 2, j h+h / 2], x_{2} \in(k h-h / 2, k h+h / 2]$, and $y_{2} \in(l h-h / 2, l h+h / 2]$ for some $i, j, k, l$. Then

$$
f_{\approx}\left(x_{1}, y_{1}\right)+f_{\widetilde{B}}\left(x_{2}, y_{2}\right)=f_{\widetilde{A}}(i h, j h)+f_{\widetilde{B}}(k h, l h) .
$$

Hence,

$$
\max _{\substack{x_{1}+x_{2}=m h \\ y_{1}+y_{2}=n h}}\left(f_{\approx}\left(x_{1}, y_{1}\right)+f_{\widetilde{B}}\left(x_{2}, y_{2}\right)\right)=\max _{\substack{i+k=m \\ j+l=n}}\left(f_{\approx}(i h, j h)+f_{\tilde{B}}(k h, l h)\right) .
$$

On other side,

$$
\begin{aligned}
& H T(\widetilde{\widetilde{A}})+H T(\widetilde{\widetilde{B}})=\bigcup_{i j} d\left[i h, j h, f_{\approx}(i h, j h)\right]+\bigcup_{k l} d\left[k h, l h, f_{\widetilde{B}}(k h, l h)\right]= \\
& \bigcup_{i j k l}\left(d\left[(i+k) h,(j+l) h, f_{A}(i h, j h)+f_{B}(k h, l h)\right]\right)=\bigcup_{m n} d\left[m h, n h, \max _{\substack{i+k=m \\
j+l=n}}\left(f_{\mathcal{A}}(i h, j h)+f_{B}(k h, l h)\right)\right] .
\end{aligned}
$$

Hence,

$$
H T(\widetilde{\widetilde{A}})+H T(\widetilde{\widetilde{B}})=H T(\widetilde{\widetilde{A}}+\widetilde{\widetilde{B}})
$$


It follows from the lemma proved that

$$
\lim _{h \rightarrow 0} H T(A+B)=\lim _{h \rightarrow 0}(H T(A)+H T(B)) .
$$

It can also be shown that

$$
H T(\tilde{\widetilde{A}}-\tilde{\widetilde{B}})=\left(H T(\tilde{\widetilde{A}})^{c}+H T(\tilde{\widetilde{B}})\right)^{c},
$$

provided $f_{A}(x, y) \geq f_{B}(x, y)$, where $A^{c}$ means the complement of the figure $A$ and $X-Y$ means Minkowski difference.

\section{On possible applications of the commutative properties of halftoning}

As is known, manipulating 3-dimensional objects is an important problem in many practical applications ranging from solid modeling in Computer Aided Design to mobile vehicle motion planning amidst obstacles and simulation of moving through virtual worlds. Such manipulations of 3-D objects are often reduced to performing set operations on them and to computing their Minkowski sums and differences (i.e. dilations and erosions respectively).

However, the latter operations are very time consuming and have, for example, $O(N M)$ time complexity even for 3-D polyhedrons in vector representation, where $N$ and $M$ are numbers of vertices of polyhedrons to be intersected, united, added, or subtracted $[\mathrm{P}]$.

That is why during the last decade there was significant interest in implementation of these operations optically because these operations on 2-D figures can be performed in constant time [CB, CAK, LKKE]. However, it was not clear how to implement these operations on 3-D figures optically.

The commutative properties of halftoning, proven above, show a way to optical implementation of set operations and Minkowski additions and subtractions on 3-D objects. Specifically, an algorithm for manipulating 3-D objects optically in constant time looks as follows:

Step 1. Encode 3-D objects $A$ and $B$ to be manipulated as 2-D gray scale images. Specifically, an object $A=\left\{(x, y, z) \mid z=f_{A}(x, y)\right\}$ is encoded as a planar gray scale image whose gray level at a point $(x, y)$ is equal to $f_{A}(x, y)$. In what follows we denote such a gray scale representation of objects $A$ and $B$ as $G S(A)$ and $G S(B)$ repectively.

Step 2. Perform halftoning of $G S(A)$ and $G S(B)$ and thereby obtain images $H T(A)$ and $H T(B)$ respectively.

Step 3. Compute either $H T(A) \bigcup H T(B)$, or $H T(A) \cap H T(B)$, or $H T(A) \pm$ $\overline{H T(B)}$ depending on the request, and thereby obtain either $H T(A \cup B)$, or $H T(A \bigcap B)$, or some approximation of $H T(A \pm B)$ respectively, so that approximation of $H T(A \pm B)$ can be obtained to within any choosen in advance degree of accuracy. 
Step 4. Convert $H T(A \bigcup B), H T(A \cap B)$, or $H T(A \pm B)$ into $G S(A \cup B)$, $\overline{G S}(A \cap B)$, or $G S(A \pm B)$ respectively and thereby obtain a planar gray scale representation of the results of the above operations on 3-D objects $A$ and $B$.

Since, the gray scale representation can be converted at Step 2 into the halftone representation optically in constant time [B], and, since, the operations $U, \cap,+$, and - on 2-dimensional images at Step 3 can be also performed optically in constant time [CB], we obtain:

Theorem 3. Union, intersection, dilation (Minkowski sum), and erosion (Minkowski difference) of two 3-dimensional images can be (approximately) computed optically in constant time to within any degree of accuracy chosen in advance.

The theorem just stated paves the way to implementation of the 3-D binary image algebra optically.

The commutative properties of halftoning with respect to set and morphological operations may also have other applications.

\section{Conclusion}

We investigated the interaction between two important tools for image processing - halftoning and the binary image algebra. We found that halftoning commutes with such basic operations of the binary image algebra as union, intersection, Minkowski sum and difference. It immediately implied the possibility of manipulating 3-D images optically in constant time.

I belive that practical consequences of this marriage of halftoning and the binary image algebra will not be too long in coming.

\section{References}

[B] O. Bryngdahl, Halftone images: spatial resolution and tone reproduction, Journal of Optical Society of America, 68, pp. 416 (1978).

[CAK] A. K. Cherri, A. A. S. Awwal, M. A. Karim, Morphological transformations based on optical symbolic substitution and polarization-encoded optical shadowcasting systems, Optics Communications, 82(5,6), $441-445$ (1991).

[CB] D. Casasent, E. C. Botha, Optical symbolic substitution for morphological transformations, Applied Optics, 27(18), 3806 - 3810 (1988).

[DS] S. R. Dashiell, A. A. Sawchuk, Nonlinear optical processing: nonmonotonic halftone cells and phase halftones, Applied Optics, 16(7), 1936- 1943 (1977).

[HJS] K. S. Huang, B. K. Jenkins, A. A. Sawchuk, Binary image algebra and optical cellular logic processor design, Computer Vision, Graphics, and Image Processing, 45, 295-345 (1989).

[KG] H. Kato, J. W. Goodman, Nonlinear filtering in coherent optical systems through halftone screen processes, Applied Optics, 14(8), 1813-1824 (1975).

[L] H. K. Liu, Coherent optical analog-to-digital conversion using a single halftone photograph, Applied Optics, 17(14), 2181- 2185 (1978). 
[LKKE] Y. Li, A. Kostrzewski, D. H. Kim, G. Eichmann, Compact parallel real-time programmable optical morphological image processor, Optics Letters, 14(18), 981 - 983 (1989).

[P] F. Preparata, M. Shamos, Computational geometry: An Introduction, SpringerVerlag, 1985. 